\title{
Parallel and Series Connection of Dark Electric Current in Liquid and Second Law of Thermodynamics
}

\author{
Sergey A. Gerasimov
}

\section{ABSTRACT}

Liquid in contact with two asymmetrical spiral-shape aluminum electrodes behaves like a still weak source of electrical energy. Almost the only way to increase the efficiency of such a source of electrical energy is to reduce internal resistance. Reducing internal resistance is equivalent to using multiple sources of electrical energy connected in series or in parallel. To check this for such unusual sources it is first necessary to study the properties of each source, which is the voltage drop across the load resistance and the internal resistance of each source. Detailed analysis of experimental data shows that the process of forming the dark current is different from a chemical one.

Keywords: second law of thermodynamics, electric current, electric energy, internal resistance, voltage.

Published Online: March 26, 2021

ISSN: $2684-4451$

DOI :10.24018/ejphysics.2021.3.2.57

\section{S. A. Gerasimov}

Department of General Physics, Faculty

of Physics, Southern Federal

University, Russian Federation.

(e-mail: gsim1953@mail.ru)

*Corresponding Author

\section{INTRODUCTION}

In physics and in electronic engineering, dark current is the relatively small electric current that flows through photosensitive devices such as a photomultiplier tube or photodiode even when no photons are entering the device. It consists of the charges generated in the detector when no outside radiation is entering the detector. Physically, dark current is due to the random generation of electrons and holes within the depletion region of the device [1]. If voltage applied to two electrodes of such devices, value of the dark electric is typically $10^{-7} \mathrm{~A}$. Another thing if a photosensitive device consists of two aluminum electrodes immersed in water. In this case the value of electric current can achieve $0.1 \mathrm{~mA}$ without external voltage [2].

The fact that the dark electric current flows without weakening for a very long time, weeks and even months [3], allows us to consider it a source of electrical energy. The energetic properties of such an unusual source of electrical energy must be studied. This primarily refers to internal resistance. It is known that parallel connection of conventional sources reduces internal resistance. The originality of the subject of study can lead to its unusual properties. A detailed consideration of the series connection of two cells with aluminum spiral-like electrodes immersed in distilled water will not be a hindrance. This connection can significantly increase the voltage drop across the load resistance.

At normal temperatures, aluminum does not chemically react with distilled water at all. This is prevented by a thin oxide layer on the aluminum surface. On the other hand, the oxide film is so thin that it makes possible the tunneling effect, as a result of which charged particles pass from one medium to another [4,5]. Since the effect is quantum, the second law of thermodynamics does not have to be fulfilled
[6]. Despite the quantum nature of the phenomenon, the corresponding electric current can reach $1 \mathrm{~mA}$ or more [7]. In any case, the nature of the electric current flowing in the circuit for a very long time without visible external influence must be found out. A simple, reliable and accurate way to do this is to study the series and parallel connection of multiple sources of the dark electric current.

\section{APPARATUS AND MEASUREMENTS}

Two approximately identical aluminum spirals $S$ with average diameter $70 \mathrm{~mm}$ and with step between wraps of $7 \mathrm{~mm}$ and two approximately identical aluminum cylindrical electrodes $C$ with diameter $7 \mathrm{~mm}$ immersed in distilled water $L$ form the basis of each of the two sources of dark current (Fig. 1).

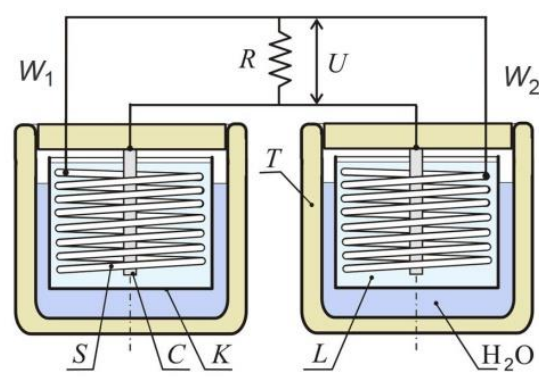

Fig. 1. Immersed in distilled water $L$ spiral-shape electrodes $S$ and cylindrical electrodes $C$ connected in parallel.

The diameter of aluminum wire of the aluminum spiralshape electrode is $4 \mathrm{~mm}$, the high of the spiral is $70 \mathrm{~mm}$. The depth of immersion of electrodes was $6 \mathrm{~cm}$. With this choice of materials chemical processes are almost completely suppressed. 
Cells $K$ with electrodes and distilled water are kept in thermostats $T$ with ordinary water. This is done in order to maximally exclude the influence of ambient temperature fluctuations on the results of measuring the voltage on the load resistor $R$.

In order to determine the electromotive force and internal resistance corresponding to parallel or series connection one should measure these values $E_{i}$ and $r_{i}$ for each source $W_{1}$ and $W_{2}$.

Remembering that

$$
U_{i}=\frac{E_{i}}{R+r_{i}} R
$$

one can rewrite this equation in the form

$$
1 / U_{i}=1 / E_{i}+\left(r_{i} / E_{i}\right) / R
$$

convenient for linear approximation.

This approach turned out to be successful and made it possible to find the values of $E_{i}$ and $r_{\mathrm{i}}$ for each of the two sources separately (Fig. 2). Experimental data on the inverse values of reverse voltages $1 / U$ from the inverse resistance $1 / R$ values are described by dependence (1) with a sufficiently high accuracy.

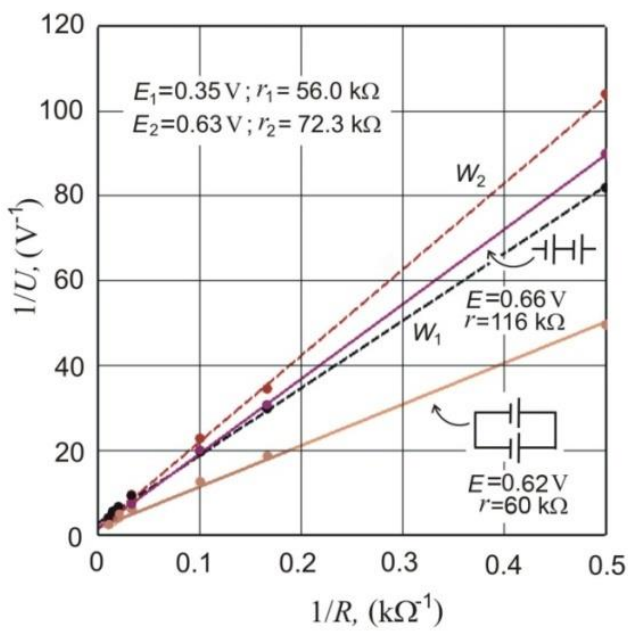

Fig. 2. Voltages $U$ at various load resistance $R$ for cells $W_{1}$ and $W_{2}$ and for series and parallel connections.

The same can be done with similar data for parallel and serial connections. Such results are also shown in Fig. 2. Series connection turned out to increase the electromotive force not significantly while the internal resistance is close to the sum $r_{1}+r_{2}$. Parallel connection practically does not change either one or the other. This is a very unusual result, so a detailed analysis of the experimental results obtained should be continued. On the one hand, parallel and series connections work as a source of electric current, and on the other, their properties are far from chemical one.

Unfortunately, the result written out in Fig. 2 is specific to that specific pair of sources with given specific dimensions and parameters. An answer has not been received to the question of what the electromotive force should be. It is not clear to what value the internal resistance can be reduced when connected in parallel.

\section{ALternative RESUlTS AND FEATURES}

For experimentally determined parameters $E_{i}$ and $r_{i}$, one can calculate the voltage on the resistor $R$ in parallel connection:

$$
U_{p}=\frac{E_{1} r_{2}+E_{2} r_{1}}{R\left(r_{1}+r_{2}\right)+r_{1} r_{2}} R
$$

This means that the electromotive force $E_{p}$ and internal resistance $r_{p}$ characterizing the parallel connection must be:

$$
E_{p}=\frac{E_{1} r_{2}+E_{2} r_{1}}{r_{1}+r_{2}},
$$

$r_{p}=\frac{r_{1} r_{2}}{r_{1}+r_{2}}$

Note, first of all, that the relation (3) is valid for independent sources of electric current, when electric current created by one source does not influence on that created by another.

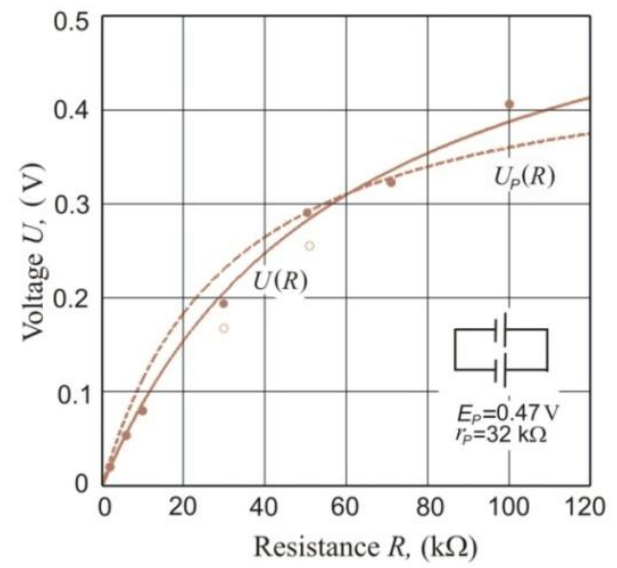

Fig. 3. Dependencies of voltage $U$ versus resistance $R$ for experimental data (points and solid line) and relation (5) (dashed line).

Substituting the experimental values of $E_{1}, E_{2}, r_{1}, r_{2}$ into (4) and (5), one obtains the electromotive force $E_{p}$ and the internal resistance $r_{p}$ near to the experimental magnitudes $E$ and $r$ rewritten in Fig. 2. The resistance dependencies $E(R)$ and $E_{p}(R)$ are also similar for the parallel connection (Fig. 3)

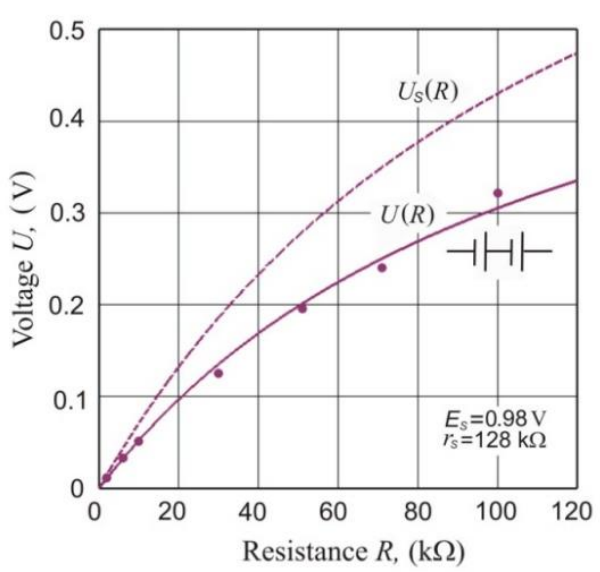

Fig. 4. Series connection of $W_{1}$ and $W_{2}$. Points and solid line are experimental results, dashed line is relation (6) 
Therefore, it is hoped that the parallel connection will improve the efficiency of such a power source, but all one can hope for is a halving of internal resistance.

For connection of sources in series, the voltage on the load resistor is:

$$
U_{s}=\frac{E_{1}+E_{2}}{R+r_{1}+r_{2}} R,
$$

that means

$$
E_{s}=E_{1}+E_{2}
$$

and

$$
r_{s}=r_{1}+r_{2}
$$

These simplest calculations are rewritten in Fig. 4. The calculated value of $E_{s}$ exceeds the experimental one shown in Fig. 2 by about one and a half times, while the calculated value of $r_{s}$ is close to the experimental magnitude of $r$.

Despite the accuracy of the experimental data plotted in Fig. 4, the dependence $U_{s}(R)$ differs significantly from $U(R)$. This does not happen with chemical sources of electric current.

\section{CONCLUSION}

The following happens. Contact of two asymmetrical aluminum electrodes with liquid behaves like a source of electric current. Parallel connection of two such sources improve the efficiency of such a combined source of electrical energy, but not significantly. When two sources are connected in series, the experimental value of the electromotive force is noticeably less than their sum. Entropy is not equal to the sum of entropies, which is an indirect confirmation of the violation of the second law of thermodynamics for an open system. So far, this is only a hypothesis that requires confirmation.

\section{REFERENCES}

[1] A. Van der Zeil, Noise in Measurements. New York.: Wiley, 1976.

[2] S.A. Gerasimov, "Photoeffect and head component of electric current in liquid," Engineering Physics, no 4, pp. 23-26, 2013.

[3] S.A. Gerasimov, "Time and temperature variations of dark electric current in liquid", American Journal of Engineering Research, vol. 9, no 11, pp. 19-23, 2020.

[4] Yu.I. Dakhnovskii, and V.V. Nefedova, "Tunneling in an Anharmonic Liquid," Soviet Physics JETF, vol. 73, no 3, pp. 492-496, 1991.

[5] S.A. Gerasimov, "Tunnel Photoelectric Effect in Liquid," Engineering Physics, no 11, pp. 3-7, 2017

[6] V. Capek, and J. Bok, "Violation of the Second Law of Thermodynamics in Quantum Microworld," Physics A, vol. 290, pp. 379-401, 2001.

[7] S.A. Gerasimov, "Electromotive force and internal resistance of dark electric current in liquid", IOSR Journal of Electrical and Electronics Engineering, vol. 15, no 3, pp. 50-55, 2020.

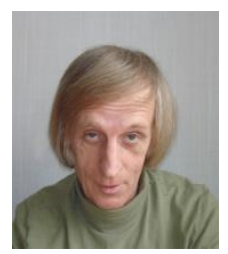

Sergey A. Gerasimov is an Associate Professor of Faculty of Physics of Southern Federal University. His research interests include Mechanics and Electrodynamics. He received $\mathrm{PhD}$ degree in Physics and Mathematics from Rostov State University, Rostov-on-Don, Russia. He has published about 200 scientific papers in International and Russian journals. He has an experience in the academic field lecturing for theoretical and experimental courses. 